\title{
Consensus on the Development of Vaccines against Naturally Acquired Melioidosis
}

\author{
Direk Limmathurotsakul, Simon G.P. Funnell, \\ Alfredo G. Torres, Lisa A. Morici, Paul J. Brett, \\ Susanna Dunachie, Timothy Atkins, \\ Daniel M. Altmann, Gregory Bancroft, \\ Sharon J. Peacock, for The Steering Group on \\ Melioidosis Vaccine Development ${ }^{1}$
}

Several candidates for a vaccine against Burkholderia pseudomallei, the causal bacterium of melioidosis, have been developed, and a rational approach is now needed to select and advance candidates for testing in relevant nonhuman primate models and in human clinical trials. Development of such a vaccine was the topic of a meeting in the United Kingdom in March 2014 attended by international candidate vaccine developers, researchers, and government health officials. The focus of the meeting was advancement of vaccines for prevention of natural infection, rather than for protection from the organism's known potential for use as a biological weapon. A direct comparison of candidate vaccines in well-characterized mouse models was proposed. Knowledge gaps requiring further research were identified. Recommendations were made to accelerate the development of an effective vaccine against melioidosis.

$\mathrm{M}$ elioidosis vaccines are urgently needed for both public health and biodefense purposes. The bacterium Burkholderia pseudomallei, the causal organism of melioidosis, was designated as a category B select agent by the US Centers for Disease Control and Prevention (CDC) and as a Tier 1 select agent by National Select Agent Registry (NASR), a joint program of the CDC and the Animal and Plant Health Inspection Service of the US Department of Agriculture. These designations have led to considerable research funding to develop a melioidosis vaccine $(1,2)$.

Author affiliations: Faculty of Tropical Medicine, Mahidol

University, Thailand (D. Limmathurotsakul); Public Health

England, London, United Kingdom (S.G.P. Funnell); University

of Texas Medical Branch, Galveston, Texas, USA (A.G. Torres);

Tulane University School of Medicine, New Orleans, Louisiana,

USA (L.A. Morici); University of South Alabama, Mobile, Alabama,

USA (P.J. Brett); University of Oxford, Oxford, United Kingdom

(S. Dunnachie); Defence Science and Technology Laboratory,

Porton Down, United Kingdom (T. Atkins); Imperial College,

London (D. M. Altmann); London School of Hygiene and Tropical

Medicine, London (G. Bancroft); University of Cambridge,

Cambridge, United Kingdom (S.J. Peacock)

DOI: http://dx.doi.org/10.3201/eid2106.141480
The potential for humans to be infected with B. pseudomallei by inhalation, the low infective dose by this route, and difficulties associated with diagnosis and treatment have led to this organism being considered to be at high risk for deliberate misuse as a weapon $(3,4,5)$.

B. pseudomallei is readily isolated from soil and water in many tropical areas across Southeast Asia, the Indian subcontinent, northern Australia, and parts of South America and the Caribbean (6). In these areas, melioidosis can be naturally acquired by skin inoculation, ingestion, and inhalation of environmental B. pseudomallei. In northeast Thailand, melioidosis kills an estimated $>1,000$ persons per year (7). A vaccine could be cost-effective for the prevention of melioidosis among populations at high risk for infection by B. pseudomallei, such as persons who have diabetes (8).

Establishing a process for selecting, assessing, and advancing potential vaccine candidates is a critical issue for the melioidosis research community. Several vaccine candidates have been shown to provide partial protection in murine models of infection $(8-10)$, but none have been tested to date in nonhuman primates (NHP) or humans. Possible candidates include live attenuated, whole-cell killed, subunit, glycoconjugate, outer membrane vesicle, plasmid DNA, and dendritic cell vaccines (8); possible candidates and candidate developments have been systematically reviewed and reported (8-10). A notable lack of standardization of protocols has resulted in variability in the animal models used (mice [BALB/c, C57BL/6, and Porton] and Syrian hamster); route of inoculation (intraperitoneal, intravenous, intranasal, intradermal, and subcutaneous); challenge strains (K96243, 1026b, 576, NCTC13392, NCTC4845, and NCTC13179); route of B. pseudomallei challenge (intraperitoneal, intravenous, aerosol inhalation, and intranasal); challenge dose; and duration of the followup period for assessing mortality rates among acute and chronic challenge models ( 5 days to 5 months) $(8-10)$. As a result of the lack of consistency in use of specific methods on specific models, it is not possible to determine the comparative efficacy of different vaccine candidates. This inconsistency prevents researchers and officials in sponsoring and funding agencies from deciding which candidates should be advanced to NHP models. Recognizing these problems, our objectives were to assemble an expert group and arrive at consensus opinions and recommendations on candidate vaccines and animal models based on currently available evidence $(8-10)$.

${ }^{1}$ Group members are listed at the end of this article. 


\section{The Working Group}

The Steering Group on Melioidosis Vaccine Development (SGMVD) was conceptualized during attendance of the VIIth World Melioidosis Congress (WMC), held in Bangkok, Thailand in September 2013. A questionnaire was initially composed by D.L. and S.J.P. based on perceived uncertainties and was intended to generate discussions regarding these barriers to progress (online Technical Appendix, http://wwwnc.cdc.gov/EID/article/21/6/14-1480Techapp1.pdf). The questionnaire was circulated to all members of the steering group in October 2013. Responses were collated and recirculated to all members during January 2014. All members of SGMVD returned the questionnaire. Then, additional experts from the United Kingdom and United States were invited to a face-to-face meeting of the steering group, which was held in March 2014 at Public Health England (PHE), Porton Down, United Kingdom. All members except Bart Currie attended the face-to-face meeting. Recommendations from the SGMVD were circulated for approval. All members agreed with the consensus opinions of the meeting attendees.

\section{Development of Melioidosis Vaccines to Prevent Natural Infection Versus Biodefense Use}

The development process of a melioidosis vaccine that prevents naturally acquired infection is likely to be different than that for biodefense purposes (Table). This difference is because a biodefense vaccine is intended to protect healthy persons from inhalational inoculation, whereas a vaccine against natural infection will be required to protect immune-compromised hosts, such as persons with diabetes, who comprise the highest risk group and are most often infected by skin inoculation (8). Therefore, the route of challenge in animal models should differ: inhalation should be the focus for biodefense vaccine development, and subcutaneous inoculation for natural infection vaccine development. It is also important to consider whether diabetic mouse models should be included for the evaluation of public health vaccines, and if so, which of the available diabetic mouse models best reflects the increased susceptibility of the at-risk human population. An additional consideration is whether postexposure prophylaxis with antimicrobial drugs should be included in models for biodefense vaccines. Sharing of information between different groups would enhance progress. Because the development of biodefense vaccines and therapeutics is already supported by other groups (e.g., Defense Threat Reduction Agency, National Institute of Allergy and Infectious Disease, and Biomedical Advanced Research and Development Authority [BARDA]), this process was not discussed further by the group $(8-11)$. The SGMVD agreed to focus further discussions on melioidosis vaccines that prevent naturally acquired infection.

\section{Potential Use of Melioidosis Vaccines to Prevent Natural Infection in Melioidosis-Endemic Areas}

During a discussion of needs for vaccines against naturally acquired melioidosis, steering group member Charung Muangchana, director of National Vaccine Institute (NVI), Ministry of Public Health, Thailand, supported the public health need for a melioidosis vaccine in Thailand. The point was made that no specific exclusion criteria would be proposed at the outset by NVI; for example, killed wholecell vaccines should not be excluded as a potential melioidosis vaccine to be used for public health purposes in Thailand. A killed whole-cell vaccine could be considered if it passed phases I, II, and III clinical studies; had minimal side effects; and proved to be a cost-effective intervention in Thailand.

None of the candidate vaccines tested to date have provided sterilizing immunity, but it was proposed during discussion that this should not be a barrier to using the best candidates in trials using NHP models. A melioidosis vaccine could be a cost-effective intervention for public health in areas to which melioidosis is endemic even if it only provided partial protection, resulting in reduction in disease severity and death rates among infected persons (8). For example, a melioidosis vaccine that reduced the

\begin{tabular}{|c|c|c|}
\hline Vaccine characteristics & $\begin{array}{c}\text { Vaccines against naturally acquired } \\
\text { melioidosis }\end{array}$ & $\begin{array}{l}\text { Melioidosis vaccines for } \\
\text { biodefense purposes }\end{array}$ \\
\hline \multicolumn{3}{|l|}{ Target population } \\
\hline Character & Persons with diabetes mellitus & Healthy persons \\
\hline Route of exposure & Skin inoculation & Inhalation \\
\hline \multicolumn{3}{|l|}{ Potential use of vaccine } \\
\hline Prophylaxis & Yes & Yes \\
\hline $\begin{array}{l}\text { Prophylaxis plus postexposure antimicrobial drug } \\
\text { administration }\end{array}$ & Yes* & Yest \\
\hline \multicolumn{3}{|l|}{ Animal models } \\
\hline Addition of diabetic mouse model & Required & Not required \\
\hline Route of Burkholderia pseudomallei challenge & Subcutaneous & Inhalation \\
\hline Nonhuman primate model & Required & Required \\
\hline
\end{tabular}

${ }^{*}$ Administration of antimicrobial drugs after symptoms occur.

†Administration of antimicrobial drugs after release. 
incidence of melioidosis by $25 \%$, reduced the case-fatality rate by $25 \%$, provided protection for 3 years, and cost $<100$ Thai Baht $(\approx \$ 3$ US) per course would be cost effective for a high-risk population in Thailand (estimated for population of persons who have diabetes, at a baseline annual incidence rate of 150 cases per 100,000 persons per year, and a fatality rate of $40 \%)(8)$.

\section{Process of Candidate Selection}

The SGMVD recommended that the approach used to select, develop, and clinically evaluate tuberculosis (TB) vaccine candidates (12) be used as an example for a gating process for melioidosis candidate selection, because the challenges of vaccine development for TB and melioidosis are similar. No melioidosis vaccine has been tested in humans, and much could be learned from the TB vaccine testing process. The SGMVD agreed that a head-to-head comparison of candidate vaccines in standardized mouse models would support decision making by developers, sponsors, funders, and policy makers concerning the choice of candidate vaccine(s) advanced for testing in NHP models. To reduce any potential bias in candidate selection and conflicts of interest, SGMVD agreed that the head-to-head comparison should be performed in an institution that is independent of the vaccine developer. The gateway system and background information requested is similar to those features in the process of candidate selection of other vaccines (12). The candidate vaccine developer will also be responsible for the preparation of a trial lot to be tested at an independent institute (12). These processes are similar to that being conducted for TB vaccine candidate evaluation (12).

The SGMVD noted that the information obtained from the head-to-head mouse model may not necessarily reflect the outcome that will be seen in a NHP model and eventually in human clinical trials. The purpose of using a combination of mouse and NHP models is to carefully select the optimal candidate vaccines for future clinical studies.

\section{Standardized Mouse Models for Head-to- Head Vaccine Candidate Comparison}

\section{Mouse Models}

The SGMVD recommended that 2 mouse models (BALB/c and $\mathrm{C} 57 \mathrm{BL} / 6$ ) be used for the head-to-head candidate vaccine assessment. These models were selected because they exhibit differential susceptibilities to $B$. pseudomallei infection: C57BL/6 mice are more resistant $(13,14)$. The evaluation should focus on protection from the acute stage of infection in both mouse models, reflecting the need to protect humans from rapidly fatal disease.

The SGMVD recommended that a diabetic mouse model should also be considered for use during the head- to-head vaccine assessment. However, inherent problems were recognized, and a specific diabetic mouse model was not selected because of the current paucity of information on melioidosis in diabetic mouse models.

\section{Formulas, Dosages, and Routes of Immunization}

The SGMVD agreed that the production of the candidate vaccine, formulation, adjuvants used, toxicity, immunogenicity, recommendations for dosage, and routes of immunization are the responsibility of the developers. During the head-to-head comparison, the study institute should administer the candidate vaccine according to the guidelines provided by the developers.

\section{Control Groups}

The SGMVD recommended the use of 2 control groups in each head-to-head comparison: a negative-control nonvaccinated group and a positive-control immunized group. The nonvaccinated group acts as a control for mortality outcome. The positive-control immunized group provides a benchmark for partial protection of a known vaccine candidate. The live attenuated mutant B. pseudomallei $i l v I$ (commonly referred to as $2 \mathrm{D} 2$ ) is proposed as a positive control for the positive-control immunized group on the basis of high efficacy of 2D2 $(15,16)$. This method has been tested in 2 independent laboratories in the United Kingdom $(15,16)$, is effective in both genetically susceptible (BALB/c) and resistant (C57BL/6) mouse strains, and is one of the most protective vaccines to be tested in the Burkholderia research field. It is easy to prepare as a standardized batch reagent (albeit under biosafety level 3 conditions) for use as a reference for the effectiveness of other vaccine candidates. Although this method has not been tested for efficacy against a wide variety of B. pseudomallei strains, it would be predicted to provide cross-protection on the basis that it is a live attenuated vaccine and will be expressing a range of antigens in the host. Confirming this efficacy against a chosen panel of approved B. pseudomallei challenge strains represents a crucial and feasible first step for its inclusion as a reference standard in future vaccine development programs.

\section{Challenge Strains of $B$. pseudomallei}

The SGMVD proposed the use of 2 or 3 bacterial strains selected from a standard set of $B$. pseudomallei isolates characterized and recommended by BARDA for headto-head vaccine candidate comparisons, rather than a single randomly selected strain (11). This choice was made because there is clear evidence for strain-to-strain variation in virulence of $B$. pseudomallei, which can be further confounded by the number of strain passages and quality of bacterial storage. Six strains are recommended for initial consideration: MSHR668, MSHR305, 1026b, 
1106a, K96243, and 406a. Each of the 6 strains prepared by BARDA has extensive and complete information, including source, passage history, virulence, genotype, and phenotype, and all are available from BARDA (11). Refining the recommendation for a subset of these 6 strains could be made after more data from mouse models are available.

\section{Challenge Route of $B$. pseudomallei}

To replicate the most common route of natural melioidosis infection in humans, the SGMVD suggested that the subcutaneous route be used for the challenge dose of $B$. pseudomallei. Current knowledge on mouse models challenged via the subcutaneous route of $B$. pseudomallei is limited (17), and further studies are required to provide baseline data. No vaccine candidate to date has been evaluated for efficacy against subcutaneous inoculation with $B$. pseudomallei $(8-10)$. The SGMVD proposed that baseline information should be generated for each of the 6 strains recommended by BARDA in BALB/c and $\mathrm{C} 57 \mathrm{BL} / 6$ mice by using the subcutaneous route.

\section{Challenge Dose of $B$. pseudomallei}

Because the aim of the head-to-head comparison is to compare the efficacy between $\geq 2$ vaccine candidates, the SGMVD proposed that the challenge dose of B. pseudomallei be adjusted so that all animals in the negative-control nonvaccinated group reach humane euthanasia endpoints at 7-14 days postchallenge. If the challenge dose of $B$. pseudomallei is too high, both groups could reach euthanasia endpoints too quickly, and the protective efficacy in vaccinated groups may not be evident. This practice was observed in a study evaluating vaccine candidates in protection against airborne challenge, in which all animals in the negative-control (nonvaccinated) group died by day 4 and all animals in the vaccinated groups died by day 5 (18). Alternatively, if the challenge dose of B. pseudomallei is too low, only a fraction of the nonvaccinated control groups may reach euthanasia endpoints, and the power to detect protective efficacy in vaccinated groups will be very low in a survival analysis. This result has been commonly observed $(8-10)$ when bacterial burden of major organs has been used as a secondary endpoint to evaluate protection from subclinical infection.

\section{Timing of Challenge}

It is essential that the B. pseudomallei challenge be done no sooner than 4 weeks after the last dose of vaccine to enable residual innate immune responses to quiesce. Otherwise, the survival benefit observed in mouse models could be overestimated because of the residual short-term innate immune response rather than the adaptive immune response induced by the vaccine.

\section{Duration of Follow-up for Mortality Rate Outcome}

The SGMVD proposed that the duration of follow-up efforts should be sufficient to compare the efficacy between the candidates. The steering group suggested a follow-up period of at least 28 days after challenge.

\section{Areas Requiring Further Advancement}

Animal Melioidosis Models Using Subcutaneous Route Much remains to be learned regarding use of subcutaneous B. pseudomallei inoculation in animal models. Most of the animal models developed in the past used intraperitoneal, intravenous, or inhalational B. pseudomallei inoculation; only a small number used a subcutaneous route $(17,19-21)$. A study using goats to compare subcutaneous versus intraperitoneal inoculation showed that, in animals inoculated intraperitoneally, septicemia with multiple microabscesses developed throughout the body, whereas in animals inoculated subcutaneously, localized abscesses developed in the lungs and spleen (22). It has been shown that the dose of $B$. pseudomallei required to result in a rapidly fatal infection administered by the subcutaneous route in mice is considerably higher than that required when using the inhalational route (17). The Defence Science and Technology Laboratory (Dstl), United Kingdom, has conducted subcutaneous B. pseudomallei challenge in marmosets (Callithrix jacchus) (23), but a $50 \%$ lethal dose could not be estimated because all 14 marmosets died within 4 days of infection when the lowest dose of 26 CFU was used (23). The study shows that most marmosets challenged with subcutaneous inoculation have a systemic disease with occasional lung involvement (23).

\section{Diabetic Murine Model}

A small number of diabetic mouse models have been evaluated with $B$. pseudomallei infection, but none of the candidate vaccines have been evaluated in diabetic mice. Although streptozotocin-induced diabetic mice have been used to study a range of diseases, in 1996, Brett and Woods reported that adult streptozotocin-induced diabetic Sprague-Dawley rats were not susceptible to intraperitoneal challenge with B. pseudomallei (24). In 2011, Hodgson et al. reported the use of 8-week-old BKS.Cg-Dock $7^{m}+/+$ $L e p r^{d b} / \mathbf{J}$ mice that carried a genetic mutation in the leptin receptor $(d b / d b)$ and become insulin resistant, diabetic, and hyperglycemic (24). The study showed that diabetic mice died more rapidly than nondiabetic mice after subcutaneous challenge with $B$. pseudomallei and that macrophages from diabetic mice were unable to contain and kill B. pseudomallei (20). In 2013, Hodson et al. compared C57BL/6JDock $7^{m} \mathrm{Lepr}^{\mathrm{db} /++}$ mice to diet-induced C57BL/6 diabetic mice and found that the metabolic profiles of the 2 models were not different. The study also showed that diet-induced diabetic mice died more rapidly than did nondiabetic mice 
after subcutaneous challenge with $B$. pseudomallei and that cytokine responses were impaired in the diabetic mice (21). It has been suggested that diet-induced C57BL/6 diabetic mice might be better models than diabetic homozygotes because $d b / d b$ mice exhibit additional immune dysfunctions when compared to type 2 diabetic mice induced by a high-fat diet (21). Nonetheless, several other current and upcoming mouse models use more sophisticated strategies for gene inactivation that more closely replicate insulin resistance in type 2 diabetes (25). Further studies are needed to determine which diabetic mouse model is the most appropriate model of melioidosis in humans with diabetes and to evaluate potential candidate vaccines in that model.

\section{Nonhuman Primate Model}

It is currently unclear which NHP model is the most suitable for melioidosis vaccine evaluation. Only the marmoset has been evaluated by using a $B$. pseudomallei subcutaneous inoculation route (23). On the basis of data obtained in melioidosis inhalation models, the marmoset appears to be the most susceptible NHP; the rhesus macaque (Macaca mulat$t a$ ) is of intermediate susceptibility (26), and the cynomolgus macaque (M. fascicularis) is the most resistant to B. pseudomallei inoculation (unpub. data). The African green monkey (Chlorocebus aethiops) is reported to be comparable to the rhesus macaque in terms of susceptibility to $B$. pseudomallei when inoculated by using the inhalation route (27). Further studies are needed to determine which NHP model is the most representative of human melioidosis and will be most useful for evaluation of candidate vaccines.

\section{Immunity among Persons with Diabetes}

Immunity and response to vaccines in humans with diabetes requires further study. In the cases of influenza and hepatitis B vaccines, persons who have diabetes have comparable responses to those who do not, and persons with diabetes acquire protection against these viruses (28-31). Effectiveness of pneumococcal vaccines is also observed in persons with diabetes, particularly in preventing pneumococcal bacteremia $(32,33)$. However, the immune deficit leading to susceptibility to melioidosis in persons with diabetes has not been clearly established. The mechanisms of immune protection against melioidosis are complex and involve both innate and adaptive immunity (3). An understanding of immunologic responses among persons with diabetes against melioidosis and correlates of protection would inform the development of a melioidosis vaccine that protects against natural infection.

\section{Funding for Head-to-Head Comparison and Future Objectives of SGMVD}

The SGMVD will seek funding from multiple sources for the head-to-head comparison of candidate vaccines at an independent institute. The SGMVD aims to communicate by using email, meet yearly to steer the process of vaccine development, continue to identify knowledge gaps on vaccine development, and ensure that the head-to-head comparison is implemented and conducted within a timely fashion.

\section{Potential of Phase I, II and III Clinical Studies}

Using the stage-gating approach, the SGMVD will develop robust criteria for advancing vaccine candidates from the NHP model stage to the phase I, II, and III clinical studies. SGMVD will also consider vaccine candidates developed for biodefense purposes if any are found to be effective by other groups, including Defense Threat Reduction Agency, National Institute of Allergy and Infectious Disease, and BARDA. On the basis of preliminary discussions, candidate vaccines that pass the first head-to-head comparison in 2 different mouse models and a further diabetic mouse model should go forward to be evaluated in a NHP model. The SGMVD does not consider that a diabetic NHP model is required. The first phase I and phase II human clinical studies should initially be conducted in healthy volunteers to determine safety and immunogenicity of vaccines, and promising candidates should be examined in phase I and phase II clinical studies that include persons with diabetes.

\section{Conclusions}

The SGMVD has recognized the need for the head-to-head comparison of candidate vaccines in standardized mouse models using a defined set of criteria and an independent institute to evaluate melioidosis candidate vaccines for advancement to the NHP model stage. None of the animal models will be a perfect reflection of human infection; the main purpose of using mouse and NHP models is to carefully select the optimal candidate vaccines for future clinical studies by using a gating process approach.

The ultimate aim of the SGMVD is to facilitate the production of a vaccine to protect against naturally acquired infection, with potential to be used in high-risk groups such as persons with diabetes in areas to which melioidosis is endemic, such as northeast Thailand. The SGMVD has identified knowledge gaps in the process of advancing melioidosis vaccines to the point of early phase clinical studies. The SGMVD will also continue to seek advice from experts in related fields over time, including those with expertise in vaccine development and testing, animal models $(20,21)$, and bacterial genomics. This committee aims to hasten the processes required to obtain a cost-effective, safe, and licensed vaccine against melioidosis.

Members of the SGMVD: Direk Limmathurotsakul (Mahidol University, Thailand); Alfredo Torres (University of Texas, USA); Andrew Simpson (Public Health England, UK); Bart Currie (Menzies School of Health Research, Australia); Charung 
Muangchana (National Vaccine Institute, Thailand); Daniel M. Altmann (Imperial College, United Kingdom); Ganjana Lertmemongkolchai (Khon Kaen University, Thailand); Greg Bancroft (London School of Hygiene and Tropical Medicine, UK); Lisa A. Morici (Tulane University, USA); Paul Brett (University of South Alabama, USA); Rick Titball (University of Exeter, UK); Simon Funnell (Public Health England, UK); Susie Dunnachie (University of Oxford, UK); Tim Atkins (Defence Science and Technology Laboratory, UK); and Sharon J. Peacock (University of Cambridge, UK).

\section{Acknowledgments}

We thank Andy Scott, Ann Rawkins, Graham Hatch, Julia Vipond, Michelle, and Rosemary Boyton for participating in the meeting as invited experts and for the technical inputs they provided. We thank Public Health England, United Kingdom, for hosting the meeting and Sarah Budd for her administrative assistance. We thank Dennis Dixon, Suman Mukhopadhay and Thames Pickett for their comments and viewpoints.

The meeting was supported by the National Institutes of Health, National Institute of Allergy and Infectious Diseases, USA, and Public Health England, United Kingdom.

Dr Limmathurotsakul is an assistant professor at Faculty of Tropical Medicine, Bangkok, Thailand. His research interests currently focus on tropical infectious diseases, particularly melioidosis.

\section{References}

1. Centers for Disease Control and Prevention. Bioterrorism agents/diseases [cited 2014 Jul 14] http:/www.bt.cdc.gov/agent/ agentlist-category.asp

2. National Select Agent Registry. HHS and USDA select agents and toxins. 7 CFR Part 331, 9 CFR Part 121, and 42 CFR Part 73 [cited 2014 Jul 14]. http://www.selectagents.gov/Select $\% 20$ Agents\%20and\%20Toxins\%20Exclusions.html

3. Wiersinga WJ, Currie BJ, Peacock SJ. Melioidosis. N Engl J Med. 2012;367:1035-44. http://dx.doi.org/10.1056/NEJMra1204699

4. Hoffmaster AR, AuCoin D, Baccam P, Baggett HC, Baird R, Bhengsri S, et al. Melioidosis diagnostic workshop, 2013. Emerg Infect Dis. 2015;21. http://dx.doi.org/10.3201/eid2102.141045

5. Lipsitz R, Garges S, Aurigemma R, Baccam P, Blaney DD, Cheng AC, et al. Workshop on treatment of and postexposure prophylaxis for Burkholderia pseudomallei and B. mallei Infection, 2010.Emerg Infect Dis. 2012;18:e2. http://dx.doi.org/ 10.3201/ eid1812.120638

6. Limmathurotsakul D, Dance DA, Wuthiekanun V, Kaestli M, Mayo M, Warner J, et al. Systematic review and consensus guidelines for environmental sampling of Burkholderia pseudomallei. PLoS Negl Trop Dis. 2013;7:e2105. http://dx.doi.org/10.1371/ journal.pntd.0002105

7. Limmathurotsakul D, Wongratanacheewin S, Teerawattanasook N, Wongsuvan G, Chaisuksant S, Chetchotisakd P, et al. Increasing incidence of human melioidosis in Northeast Thailand. Am J Trop Med Hyg. 2010;82:1113-7. http://dx.doi.org/10.4269/ ajtmh.2010.10-0038

8. Peacock SJ, Limmathurotsakul D, Lubell Y, Koh GC, White LJ, Day NP, et al. Melioidosis vaccines: a systematic review and appraisal of the potential to exploit biodefense vaccines for public health purposes. PLoS Negl Trop Dis. 2012;6:e1488.

http://dx.doi.org/10.1371/journal.pntd.0001488

9. Sarkar-Tyson M, Titball RW. Progress toward development of vaccines against melioidosis: a review. Clin Ther. 2010;32:143745. http://dx.doi.org/10.1016/j.clinthera.2010.07.020

10. Patel N, Conejero L, De Reynal M, Easton A, Bancroft GJ, Titball RW. Development of vaccines against Burkholderia pseudomallei. Frontiers in microbiology. 2011;2:198. http://dx.doi.org/ 10.3389\%2Ffmicb.2011.00198

11. Van Zandt KE, Tuanyok A, Keim PS, Warren RL, Gelhaus HC. An objective approach for Burkholderia pseudomallei strain selection as challenge material for medical countermeasures efficacy testing. Frontiers in cellular and infection microbiology. 2012;2:120. http://dx.doi.org/10.3389\%2Ffcimb.2012.00120

12. Barker L, Hessel L, Walker B. Rational approach to selection and clinical development of TB vaccine candidates. Tuberculosis (Edinb). 2012;92(Suppl 1):S25-9. http://dx.doi.org/10.1016/ S1472-9792(12)70009-4

13. Liu B, Koo GC, Yap EH, Chua KL, Gan YH. Model of differential susceptibility to mucosal Burkholderia pseudomallei infection. Infect Immun. 2002;70:504-11. http://dx.doi.org/ 10.1128/IAI.70.2.504-511.2002

14. Leakey AK, Ulett GC, Hirst RG. BALB/c and C57Bl/6 mice infected with virulent Burkholderia pseudomallei provide contrasting animal models for the acute and chronic forms of human melioidosis. Microb Pathog. 1998;24:269-75. http://dx.doi.org/10.1006/ mpat.1997.0179

15. Atkins T, Prior RG, Mack K, Russell P, Nelson M, Oyston PC, et al. A mutant of Burkholderia pseudomallei, auxotrophic in the branched chain amino acid biosynthetic pathway, is attenuated and protective in a murine model of melioidosis. Infect Immun. 2002;70:5290-4. http://dx.doi.org/10.1128/IAI.70.9.5290-5294.2002

16. Haque A, Chu K, Easton A, Stevens MP, Galyov EE, Atkins T, et al. A live experimental vaccine against Burkholderia pseudomallei elicits CD4+ T cell-mediated immunity, priming T cells specific for 2 type III secretion system proteins. J Infect Dis. 2006;194:1241-8. http://dx.doi.org/10.1086/508217

17. Titball RW, Russell P, Cuccui J, Easton A, Haque A, Atkins T, et al. Burkholderia pseudomallei: animal models of infection. Trans R Soc Trop Med Hyg. 2008;102(Suppl 1):S111-6. http://dx.doi.org/10.1016/S0035-9203(08)70026-9

18. Nelson M, Prior JL, Lever MS, Jones HE, Atkins TP, Titball RW. Evaluation of lipopolysaccharide and capsular polysaccharide as subunit vaccines against experimental melioidosis. J Med Microbiol. 2004;53:1177-82. http://dx.doi.org/10.1099/ jmm.0.45766-0

19. Valade E, Thibault FM, Gauthier YP, Palencia M, Popoff MY, Vidal DR. The PmlI-PmlR quorum-sensing system in Burkholderia pseudomallei plays a key role in virulence and modulates production of the MprA protease. J Bacteriol. 2004;186:2288-94. http://dx.doi.org/10.1128/JB.186.8.2288-2294.2004

20. Hodgson KA, Morris JL, Feterl ML, Govan BL, Ketheesan N. Altered macrophage function is associated with severe Burkholderia pseudomallei infection in a murine model of type 2 diabetes. Microbes Infect. 2011;13:1177-84. http://dx.doi.org/ 10.1016/j.micinf.2011.07.008

21. Hodgson KA, Govan BL, Walduck AK, Ketheesan N, Morris JL. Impaired early cytokine responses at the site of infection in a murine model of type 2 diabetes and melioidosis comorbidity. Infect Immun. 2013;81:470-7. http://dx.doi.org/10.1128/ IAI.00930-12

22. Narita M, Loganathan P, Hussein A, Jamaluddin A, Joseph PG. Pathological changes in goats experimentally inoculated with Pseudomonas pseudomallei. Natl Inst Anim Health Q (Tokyo). 1982;22:170-9. 
23. Nelson M, Salguero FJ, Dean RE, Ngugi SA, Smither SJ, Atkins $\mathrm{TP}$, et al. Comparative experimental subcutaneous glanders and melioidosis in the common marmoset (Callithrix jacchus). Int J Exp Pathol. 2014;95:378-91. http://dx.doi.org/10.1111/ iep. 12105

24. Brett PJ, Woods DE. Structural and immunological characterization of Burkholderia pseudomallei O-polysaccharide-flagellin protein conjugates. Infect Immun. 1996;64:2824-8.

25. Plum L, Wunderlich FT, Baudler S, Krone W, Bruning JC. Transgenic and knockout mice in diabetes research: novel insights into pathophysiology, limitations, and perspectives. Physiology (Bethesda). 2005;20:152-61. http://dx.doi.org/10.1152/ physiol.00049.2004

26. Yingst SL, Facemire P, Chuvala L, Norwood D, Wolcott M, Alves DA. Pathological findings and diagnostic implications of a rhesus macaque (Macaca mulatta) model of aerosol-exposure melioidosis (Burkholderia pseudomallei). J Med Microbiol. 2014;63:118-28. http://dx.doi.org/10.1099/jmm.0.059063-0

27. Yeager JJ, Facemire P, Dabisch PA, Robinson CG, Nyakiti D, Beck K, et al. Natural history of inhalation melioidosis in rhesus macaques (Macaca mulatta) and African green monkeys (Chlorocebus aethiops). Infect Immun. 2012;80:3332-40. http://dx.doi.org/10.1128/IAI.00675-12

28. Colquhoun AJ, Nicholson KG, Botha JL, Raymond NT. Effectiveness of influenza vaccine in reducing hospital admissions in people with diabetes. Epidemiol Infect. 1997;119:335-41. http://dx.doi.org/10.1017/S095026889700825X
29. Marseglia GL, Scaramuzza A, d'Annunzio G, Comolli G, Gatti M, Lorini R. Successful immune response to a recombinant hepatitis B vaccine in young patients with insulin-dependent diabetes mellitus. Diabet Med. 1996;13:630-3. http://dx.doi.org/ $10.1002 /(\mathrm{SICI})$ 1096-9136(199607)13:7<630::AID-DIA137>3.0.CO;2-7

30. Frasca D, Diaz A, Romero M, Mendez NV, Landin AM, Ryan JG, et al. Young and elderly patients with type 2 diabetes have optimal $\mathrm{B}$ cell responses to the seasonal influenza vaccine. Vaccine. 2013;31:3603-10. http://dx.doi.org/10.1016/ j.vaccine.2013.05.003

31. Smith SA, Poland GA. Use of influenza and pneumococcal vaccines in people with diabetes. Diabetes Care. 2000;23:95-108. http://dx.doi.org/10.2337/diacare.23.1.95

32. Sisk JE, Moskowitz AJ, Whang W, Lin JD, Fedson DS, McBean AM, et al. Cost-effectiveness of vaccination against pneumococcal bacteremia among elderly people. JAMA. 1997;278:1333-9. http://dx.doi.org/10.1001/ jama.1997.03550160053038

33. Kesavadev J, Misra A, Das AK, Saboo B, Basu D, Thomas N, et al. Suggested use of vaccines in diabetes. Indian J Endocrinol Metab. 2012;16:886-93. http://dx.doi.org/10.4103/2230-8210.102982

Address for correspondence: Direk Limmathurotsakul, Faculty of Tropical Medicine, Mahidol University, 420/6 Rajvithi Road, Bangkok, 10400, Thailand; e-mail: direk@tropmedres.ac

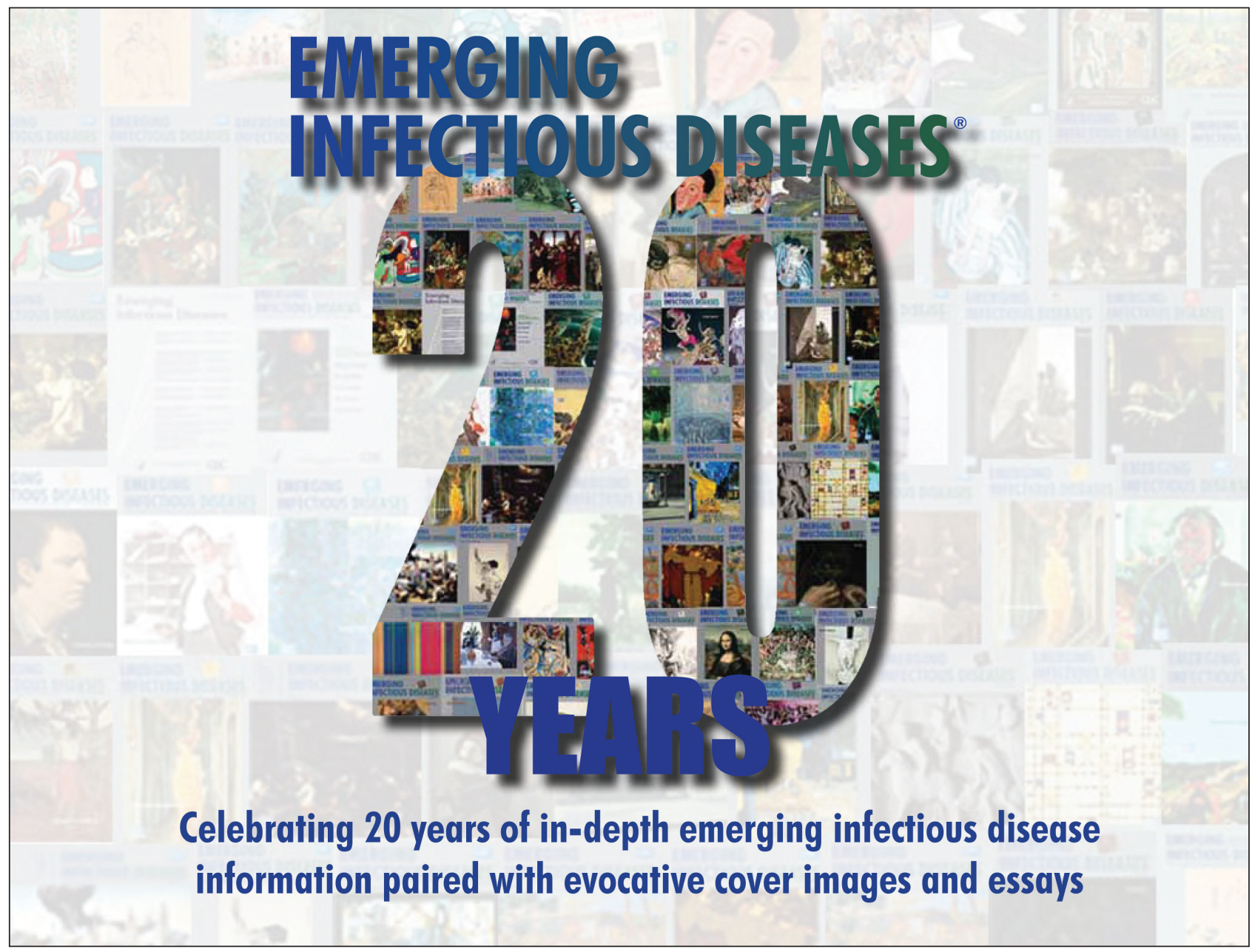

